PROCEEDINGS OF THE

AMERICAN MATHEMATICAL SOCIETY

Volume 128, Number 7, Pages 1991-2000

S 0002-9939(99)05286-7

Article electronically published on November 1, 1999

\title{
VIEWING AF-ALGEBRAS AS GRAPH ALGEBRAS
}

\author{
D. DRINEN
}

(Communicated by David R. Larson)

\begin{abstract}
Every AF-algebra $A$ arises as the $C^{*}$-algebra of a locally finite pointed directed graph in the sense of Kumjian, Pask, Raeburn, and Renault. For AF-algebras, the diagonal subalgebra defined by Strătilă and Voiculescu is consistent with Kumjian's notion of diagonal, and the groupoid arising from a well-chosen Bratteli diagram for $A$ coincides with Kumjian's twist groupoid constructed from a diagonal of $A$.
\end{abstract}

\section{INTRODUCTION}

A Bratteli diagram 22 can be viewed as a particular kind of directed graph, and to each such diagram there can be associated a unique $\mathrm{AF} C^{*}$-algebra. Recently, Kumjian, Pask, Raeburn, and Renault introduced a more general notion of constructing a $C^{*}$-algebra from a directed graph. For certain kinds of graphs, their strategy for doing so was to build from the graph a locally compact $r$-discrete groupoid, and then use the theory of Renault [8] to construct a $C^{*}$-algebra. In this paper, we show that these two procedures are compatible.

Suppose $E$ is a row-finite directed graph with no sinks. In [6], Kumjian, Pask, Raeburn, and Renault construct a locally compact $r$-discrete groupoid $\mathcal{G}_{E}$ from $E$, and study the associated $C^{*}$-algebra $C^{*}\left(\mathcal{G}_{E}\right)$. The class of $C^{*}$-algebras that arise in this way includes, up to Morita equivalence, Cuntz-Krieger algebras and AFalgebras. In [5], Kumjian, Pask, and Raeburn define the $C^{*}$-algebra $C^{*}(E)$ of any row-finite directed graph $E$, and note that $C^{*}(E)$ coincides with $C^{*}\left(\mathcal{G}_{E}\right)$ in case $E$ has no sinks. They then show that $C^{*}(E)$ is an AF-algebra if and only if $E$ has no (directed) loops. The main result of this paper is that every AF-algebra arises in this way, at least if we are willing to point the graph. That is, every AF-algebra $A$ is the $C^{*}$-algebra of a row-finite (pointed) directed graph. Not surprisingly, that directed graph will be a Bratteli diagram for $A$. An arbitrary Bratteli diagram for $A$, however, may not produce the desired result, so we start in Section 2 by giving a procedure for building appropriate Bratteli diagrams. Section 3 recalls the basics from [6] and then proves the main result.

In Section 4 we explore the notion of the diagonal subalgebra. This is defined in [9] for unital AF-algebras and in [4] for general $C^{*}$-algebras. We show that every AF-algebra (unital or not) contains a subalgebra which is diagonal in the sense of [4] and that, not surprisingly, the two notions of diagonal coincide in the AF case.

Received by the editors March 23, 1998 and, in revised form, August 18, 1998.

1991 Mathematics Subject Classification. Primary 46L05; Secondary 22A22.

(C)2000 American Mathematical Society 
Further, our construction of a groupoid from a suitable Bratteli diagram for $A$ is compatible with Kumjian's construction in [4] of a groupoid from a diagonal of $A$.

This paper was written while I was a student at Arizona State University, and it formed my masters thesis. I would like to take this opportunity to express my gratitude to my advisor, John Quigg, for all the patient help and guidance he provided. I would also like to thank Alex Kumjian, David Pask, and Jack Spielberg for their helpful discussions about the ideas in this paper.

\section{Preliminaries}

Our conventions for AF-algebras come primarily from [3]. Given a sequence of finite-dimensional $C^{*}$-algebras $\left\{A_{n}\right\}$, and injective ${ }^{*}$-homomorphisms $\phi_{n}: A_{n} \hookrightarrow$ $A_{n+1}$, we define $\lim _{\rightarrow}\left(A_{n}, \phi_{n}\right)$ to be the $C^{*}$-algebraic direct limit of the inductive system

$$
A_{1} \stackrel{\phi_{1}}{\longrightarrow} A_{2} \stackrel{\phi_{2}}{\longrightarrow} A_{3} \stackrel{\phi_{3}}{\longrightarrow} \cdots .
$$

The $C^{*}$-algebra $\lim _{\rightarrow}\left(A_{n}, \phi_{n}\right)$ is called an $A F$-algebra.

Given an AF-algebra

$$
A=\underset{\lim }{\longrightarrow}\left(A_{n}, \phi_{n}\right)
$$

let $p_{n}$ be the number of simple summands in the algebra $A_{n}$, and let $[n, 1],[n, 2], \ldots$, $\left[n, p_{n}\right]$ denote the sizes of the corresponding matrix algebras. That is, for each $n \in \mathbf{N}, A_{n}=\bigoplus_{i=1}^{p_{n}} A_{i}^{n}$, with $A_{i}^{n} \cong M_{[n, i]}$. We will denote by $\left[K^{(n)}\right]$ the $p_{n+1} \times p_{n}$ matrix of multiplicities corresponding to the map $\phi_{n}: A_{n} \rightarrow A_{n+1}$. That is, the multiplicity of the embedding $\phi_{i, j}^{n}: A_{j}^{n} \longrightarrow A_{i}^{n+1}$ is $k_{i, j}^{(n)}$. For $m<n$, define $\phi_{n, m}=\phi_{n-1} \circ \phi_{n-2} \circ \cdots \circ \phi_{m}: A_{m} \longrightarrow A_{n}$.

Given this set-up, we construct a Bratteli $\operatorname{diagram} G$ for $A$ as follows: the vertex set $V$ for $G$ is defined by $V=\bigcup_{i=1}^{\infty} V^{n}$ and $V^{n}=\left\{v_{1}^{n}, v_{2}^{n}, \ldots, v_{p_{n}}^{n}\right\}$. For every $n \in N$, and for every $i \leq p_{n+1}$ and $j \leq p_{n}$, put $k_{i, j}^{(n)}$ edges from $v_{j}^{n}$ to $v_{i}^{n+1}$. $G$ is now a row-finite (locally finite, in fact) directed graph in the sense of [6]. There are, of course, many Bratteli diagrams associated with a particular AF-algebra, but we will call the one constructed above the Bratteli diagram corresponding to the system $\left(A_{n}, \phi_{n}\right)$. One more bit of notation: for $n>1, i \leq p_{n}$, put

$$
\sigma_{i}^{n}=[n, i]-\sum_{j=1}^{p_{n-1}} k_{i, j}^{(n-1)}[n-1, j],
$$

and put $\sigma_{i}^{1}=[1, i]$ for $i \leq p_{1}$. Think of $\sigma_{i}^{n}$ as the "slack" at vertex $i$ of level $n$, the extent to which the embeddings of the algebras at level $n-1$ fail to fill up the algebra $A_{i}^{n}$. Of course $\sigma_{i}^{n}$ is a nonnegative integer for all $n, i$; it will also be convenient in what follows to arrange things so that $\sigma_{i}^{n} \leq 1$. The idea of the following lemma is to replace a Bratteli diagram which has vertices with too much slack with one that corresponds to the same AF-algebra, but which has limited slack at all of its vertices.

Lemma 1. Every AF-algebra $A=\lim _{\rightarrow}\left(A_{n}, \phi_{n}\right)$ is isomorphic to an AF-algebra $D=\lim _{\rightarrow}\left(D_{n}, \psi_{n}\right)$ which satisfies $D_{1}=\mathbf{C}$ and

$$
\sigma_{i}^{n} \leq 1 \text { for all } n>1 \text { and all } i \leq p_{n} .
$$


Before we proceed with the proof, we present an example to guide the reader. Suppose we are confronted with the following undesirable Bratteli diagram:

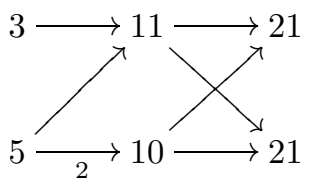

First we fix the beginning:

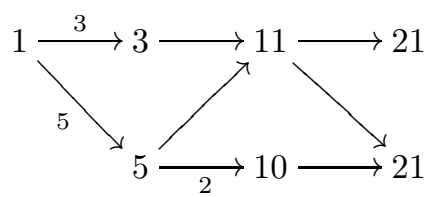

Then we add a layer:

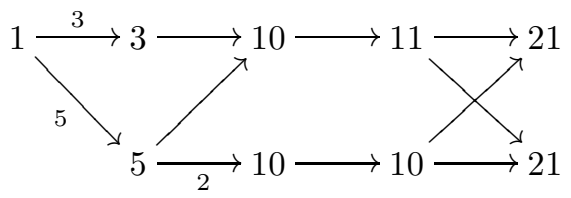

And another:

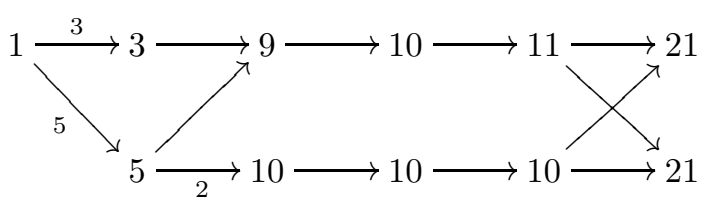

Further, we will fix the maps along the way so that, for instance, the map $M_{5} \hookrightarrow$ $M_{11}$ in the original diagram is the same as the composition of the three maps $M_{5} \hookrightarrow M_{9} \hookrightarrow M_{10} \hookrightarrow M_{11}$ in the final diagram.

Proof. Given $A$ with $A_{1} \neq \mathbf{C}$, define a new inductive system $\left(B_{n}, \rho_{n}\right)$ as follows. Let $B_{1}=\mathbf{C}$, and let $B_{n}=A_{n-1}$ for $n>1$. Define $\rho_{n}=\phi_{n-1}$ for $n>1$, and let $\rho_{1}$ be the unique unital homomorphism from $\mathbf{C}$ to $B_{2}$. If $B$ is defined to be $\lim _{\rightarrow}\left(B_{n}, \rho_{n}\right)$, then it is clear that $B \cong A$. So we may assume, without loss of generality, that $A_{1}=\mathbf{C}$. We now proceed to modify $A$ so that it satisfies condition (11).

First let $n$ be the smallest integer for which there is an $i \leq p_{n}$ with $\sigma_{i}^{n}>1$. Define $D_{j}=A_{j}$ for $j=1, \ldots, n-1$ and let $\psi_{j}=\phi_{j}$ for $j=1, \ldots, n-2$. Now define $D_{n}$ as follows. For $k=1, \ldots, p_{n}$, if $\sigma_{k}^{n} \leq 1$, put $D_{k}^{n}=A_{k}^{n}$. If $\sigma_{k}^{n}>1$, choose $D_{k}^{n}$ such that $\bigcup_{j=1}^{p_{n-1}} \operatorname{range}\left(\phi_{k, j}^{n-1}\right) \subset D_{k}^{n} \subset A_{k}^{n}$ and $D_{k}^{n} \cong M_{[n, k]-1}$. Put $D_{n}=\bigoplus_{k=1}^{p_{n}} D_{k}^{n}$.

Let $\psi_{n-1}=\phi_{n-1}$, but viewed as a map from $A_{n-1}$ to $D_{n}$. Now let $D_{n+1}=A_{n}$ and let $\psi_{n}: D_{n} \hookrightarrow D_{n+1}$ denote the inclusion map. It is clear that $\phi_{n-1}=$ $\psi_{n} \circ \psi_{n-1}$.

Now define $D_{n+j}=A_{n+j-1}$ for $j \geq 2$ and $\psi_{n+j}=\phi_{n+j-1}$ for $j \geq 1$. This gives a new inductive system, and thus a new AF-algebra. Now repeat this process as many times as necessary (possibly countably many) and denote the resulting system also by $D=\lim _{\rightarrow}\left(D_{n}, \psi_{n}\right)$. 
It is clear that $D$ satisfies the required condition, so it remains to show that $D \cong$ $A$. To that end, we note that $D=\lim _{\rightarrow}\left(D_{n_{k}}, \psi_{n_{k+1}, n_{k}}\right)$ for any subsequence $\left\{n_{k}\right\}$. By construction, $A_{1}=D_{1}$. Inductively, for $k>1$, we can choose an $n_{k}>n_{k-1}$ such that $A_{k}=D_{n_{k}}$. Because of the way the $\psi_{n}$ 's were constructed, the sequence $\left(D_{n_{k}}, \psi_{n_{k+1}, n_{k}}\right)$ coincides with the sequence $\left(A_{k}, \phi_{k}\right)$. Thus $A \cong D$.

\section{THE MAIN RESULT}

Before we state the main result, we collect some notation and basic facts from [6]. Given a directed graph $E$, let $E^{0}$ denote the set of vertices and $E^{1}$ the set of edges. For $e \in E^{1}$, let $s(e)$ and $r(e)$ denote the source and range of $e$, so $s, r: E^{1} \longrightarrow E^{0}$. For $n \in \mathbf{N}$, let $E^{n}=\left\{e_{1} e_{2} \ldots e_{n} \mid e_{i} \in E^{1}, s\left(e_{i}\right)=r\left(e_{i-1}\right), i=2, \ldots, n\right\}$, the set of all paths in $E$ of length $n$. Let $E^{*}=\bigcup_{n \in \mathbb{N}} E^{n}$, the set of all finite paths, and $E^{\infty}=\left\{e_{1} e_{2} \ldots \mid s\left(e_{i}\right)=r\left(e_{i-1}\right)\right\}$, the infinite path space of $E$.

For $x, y \in E^{\infty}, k \in \mathbf{Z}$, say $x \sim_{k} y$ if and only if $x_{i}=y_{i+k}$ for large $i$. Define $\mathcal{G}_{E}$, the path groupoid of $E$, by $\mathcal{G}_{E}=\left\{(x, k, y) \mid x \sim_{k} y\right\}$. The groupoid operations in $\mathcal{G}_{E}$ are as follows:

- $(x, k, y)^{-1}=(y,-k, x)$;

- $(x, k, y) \cdot(y, l, z)=(x, k+l, z)$.

For $\alpha, \beta \in E^{*}$ with $r(\alpha)=r(\beta)$, define $Z(\alpha, \beta)=\{(x, k, y) \mid x=\alpha z, y=\beta z, k=$ $|\alpha|-|\beta|\}$. The $Z(\alpha, \beta)$ 's are compact open $G$-sets in the topology they generate. Note that $r(x, k, y)=(x, 0, x)$, so we identify $E^{\infty}$ with $\mathcal{G}_{E}^{0}$, the unit space of $\mathcal{G}_{E}$. The relative topology on $E^{\infty}$ as a subset of $\mathcal{G}_{E}$ agrees with the product topology.

Since the $Z(\alpha, \beta)$ 's are compact and open and generate the topology of $\mathcal{G}_{E}$, we have that $C^{*}\left(\mathcal{G}_{E}\right)$ is generated by the characteristic functions of these sets. That is, $\operatorname{span}\left\{1_{Z(\alpha, \beta)} \mid \alpha, \beta \in E^{*}, r(\alpha)=r(\beta)\right\}$ is dense in $C^{*}\left(\mathcal{G}_{E}\right)$. Note how two of these characteristic functions multiply in $C^{*}\left(\mathcal{G}_{E}\right)$ :

$$
1_{Z(\alpha, \beta)} * 1_{Z(\gamma, \delta)}=\left\{\begin{array}{cl}
1_{Z(\alpha \epsilon, \delta)} & \text { if } \gamma=\beta \epsilon \\
1_{Z(\alpha, \delta \epsilon)} & \text { if } \beta=\gamma \epsilon \\
0 & \text { otherwise }
\end{array}\right.
$$

that is, the product is 0 unless one of $\beta, \gamma$ continues the other.

One more piece of notation: suppose $S$ is any subset of $E^{0}$. Considering only those paths whose source is in $S$ determines an open subset of $\mathcal{G}_{E}^{0}$. We will refer to the reduction of the groupoid $\mathcal{G}_{E}$ to this open set as $\left.\mathcal{G}_{E}\right|_{S}$, slightly abusing the notation. Distinguishing a set of vertices of the graph is known as pointing the graph. In what follows, we will be dealing with path groupoids reduced to such subsets of the unit space. Because $S$ is open, the Haar system for $\mathcal{G}_{E}$ restricts to a Haar system for $\left.\mathcal{G}_{E}\right|_{S}$. Also, $\{Z(\alpha, \beta) \mid s(\alpha), s(\beta) \in S\}$ is a base of compact open $G$-sets for the topology of $\left.\mathcal{G}_{E}\right|_{S}$, so the span of the characteristic functions of those sets is dense in $C^{*}\left(\left.\mathcal{G}_{E}\right|_{S}\right)$.

We now state the main result:

Theorem 1. Given an AF-algebra

$$
A=\underset{\lim }{\longrightarrow}\left(A_{n}, \phi_{n}\right),
$$

there is a Bratteli diagram $E$ for $A$ and a set $S$ of vertices such that $C^{*}\left(\left.\mathcal{G}_{E}\right|_{S}\right) \cong A$.

Proof. First use the above procedure (if necessary) to construct a new inductive system for the AF-algebra such that the Bratteli diagram corresponding to that 
inductive system satisfies condition (11). That is, build a Bratteli diagram for $A$ which has no excess slack. Denote by $E$ the Bratteli diagram corresponding to this inductive system. Next, define $S$ to be the set of all vertices where the slack is 1 , along with the vertex at the top level, and for each $n$ and $i$ define $F_{i}^{n}$ to be the set of all finite paths in $E$ that start in $S$ and end at the vertex $v_{i}^{n}$. That is, $S:=\left\{v_{i}^{n} \mid \sigma_{i}^{n}=1\right\} \cup\left\{v_{1}^{1}\right\}$ and $F_{i}^{n}:=\left\{\alpha \in E^{*} \mid s(\alpha) \in S, r(\alpha)=v_{i}^{n}\right\}$. Let $\left|F_{i}^{n}\right|$ denote the cardinality of $F_{i}^{n}$.

Now we claim that for every integer $n$,

$$
\left|F_{i}^{n}\right|=[n, i] \text { for every } i \leq p_{n} .
$$

The proof is by induction. First note that the case $n=1$ is taken care of by the construction of $A$. That is, since $p_{1}=1$ and $[1,1]=1$, the set $F_{1}^{1}$ has only one element, the zero-length path $v_{1}^{1}$. Now suppose that (2) is true for $n=l$. Fix $i \leq p_{l+1}$. If $\sigma_{i}^{l+1}=0$, then $v_{i}^{l+1}$ is not an element of $S$, so every path starting in $S$ and ending at $v_{i}^{l+1}$ must have come through some vertex in level $l$. There are $p_{l}$ such vertices, the $j$ th of which, by the induction hypothesis, is the range of $[l, j]$ paths emanating from $S$. There are $k_{i, j}^{(l)}$ different ways to get from $v_{j}^{l}$ to $v_{i}^{l+1}$, so we must have

$$
\left|F_{i}^{l+1}\right|=\sum_{j=1}^{p_{l}} k_{i, j}^{(l)}[l, j]=[l+1, i]-\sigma_{i}^{l+1}=[l+1, i],
$$

as desired. If $\sigma_{i}^{l+1}=1$, then the calculations are similar: we end up with $[l+1, i]-1$ paths which pass through the preceding level. Adding in the zero-length path $v_{i}^{l+1}$, which is in $S$, brings us to the right number. This establishes the claim.

Now define, for any positive integer $n$ and any $i \leq p_{n}, B_{i}^{n}:=\operatorname{span}\left\{1_{Z(\alpha, \beta)} \mid \alpha, \beta\right.$ $\left.\in F_{i}^{n}\right\} \subset C^{*}\left(\left.\mathcal{G}_{E}\right|_{S}\right)$. Note that, given two generators of $B_{i}^{n}$, say $1_{Z(\alpha, \beta)}$ and $1_{Z(\gamma, \delta)}$, because $\beta$ and $\gamma$ end at the same vertex, one cannot continue the other unless they coincide, so we have:

$$
1_{Z(\alpha, \beta)} * 1_{Z(\gamma, \delta)}=\left\{\begin{array}{cc}
1_{Z(\alpha, \delta)} & \text { if } \beta=\gamma \\
0 & \text { otherwise. }
\end{array}\right.
$$

Also, $1_{Z(\alpha, \beta)}^{*}=1_{Z(\beta, \alpha)}$. So the elements of $B_{i}^{n}$ behave like matrix units, and there are, by the above claim, $[n, i]^{2}$ of them, so $B_{i}^{n} \cong M_{[n, i]}$. Further, if $i \neq j, B_{i}^{n} B_{j}^{n}=0$ because if a path ends at $v_{i}^{n}$, it cannot be a continuation of (or be continued by) a path ending at $v_{j}^{n}$. Thus, $B_{n}:=\operatorname{span} B_{i}^{n}$ coincides with the direct sum $\bigoplus_{i=1}^{p_{n}} B_{i}^{n}$, and we have

$$
B_{n} \cong \bigoplus_{i=1}^{p_{n}} M_{[n, i]} \cong A_{n}
$$

Note now that each of these algebras embeds naturally into the next; for instance,

$$
1_{Z(\alpha, \beta)}=\sum_{\left\{e \in E^{1} \mid s(e)=r(\alpha)\right\}} 1_{Z(\alpha e, \beta e)}
$$

gives the inclusion $\iota_{n}$ of $B_{n}$ into $B_{n+1}$. The multiplicity of the embedding of $B_{j}^{n}$ into $B_{i}^{n+1}$ is the number of edges from $v_{j}^{n}$ to $v_{i}^{n+1}$, which is $k_{i, j}^{(n)}$. Thus the Bratteli diagram corresponding to $\left(B_{n}, \iota_{n}\right)$ is the same as the Bratteli diagram corresponding to $\left(A_{n}, \phi_{n}\right)$. Hence, by [2, Theorem 2.7],

$$
B:=\lim _{\rightarrow}\left(B_{n}, \iota_{n}\right) \cong A .
$$


Since $\bigcup_{n=1}^{\infty} B_{n}=\operatorname{span}\left\{1_{Z(\alpha, \beta)} \mid \alpha, \beta \in F_{i}^{n}\right.$ for some $\left.n, i\right\}$ generates $C^{*}\left(\mathcal{G}_{E} \mid{ }_{S}\right)$, it follows that $A \cong C^{*}\left(\left.\mathcal{G}_{E}\right|_{S}\right)$.

Note that it is necessary to correctly modify and correctly point the Bratteli diagram, as the following simple example shows: $M_{3}$ can be thought of as an AFalgebra with the following Bratteli diagram:

$$
1 \longrightarrow 2 \longrightarrow 3 \longrightarrow 3 \longrightarrow \cdots
$$

If we turn this into a directed graph in the obvious way and fail to point it, we obtain the following graph:

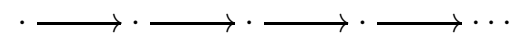

of which the $C^{*}$-algebra is $\mathcal{K}$, the compact operators. If we point the graph, for instance, at only one vertex, we obtain the graph:

$$
* \longrightarrow \cdot \longrightarrow \cdot \longrightarrow \cdot \cdots
$$

whose $C^{*}$-algebra is $\mathbf{C}$. Applying the procedure of Section 2 to the Bratteli diagram in question would produce the following pointed graph:

$$
* \longrightarrow * \longrightarrow * \longrightarrow
$$

which has the desired algebra, $M_{3}$, as a $C^{*}$-algebra.

Note that there are other procedures for modifying and pointing the Bratteli diagram that would yield the same result. One such (communicated to us by Alex Kumjian) would be to add a vertex to every level below which there is a vertex with slack greater than 0 , then add the appropriate number of edges from the new vertex to the ones with slack. That is, if $\sigma_{n, i}>0$ for some $i \leq p_{n}$, add a vertex to level $n-1$ and add $\sigma_{n, i}$ edges from the new vertex to the vertex $v_{i}^{n}$. Then point this Bratteli diagram at the added vertices. This approach is somewhat more natural because every pointed vertex is a source, but it makes for unnatural Bratteli diagrams.

Also note that, in case $A$ is unital, it is possible to construct a Bratteli diagram for $A$ which has no slack at all and which has a single vertex at the top level. In this case, it is only necessary to point the top vertex.

\section{Connection with the Diagonal}

In [4], Kumjian defines the notion of a diagonal subalgebra of a $C^{*}$-algebra $A$ as follows. An abelian subalgebra $B$ of $A$ is said to be diagonal in $A$ if it contains a positive element which is strictly positive in $A$ and if there exists a faithful conditional expectation $P: A \longrightarrow B$ such that $N_{f}(B)$ spans a dense subset of ker $P$. Here, $N_{f}(B)$ denotes the free normalizers of $B$ in $A$, namely the set of all $a \in A$ such that $a^{*} B a \cup a B a^{*} \subset B$ and $a^{2}=0$. He defines a twist as a proper Tgroupoid $\Gamma$ such that $\Gamma / \mathbf{T}$ is an $r$-discrete equivalence relation, and shows that there is a one-to-one correspondence between twists and diagonal pairs of $C^{*}$-algebras. We briefly recall the basics here.

Suppose $\Gamma$ is a twist. Define

$$
E(\Gamma)=\left\{f \in C_{c}(\Gamma) \mid f(t \gamma)=t f(\gamma) \text { for all } t \in \mathbf{T}, \gamma \in \Gamma\right\}
$$

and

$$
D(\Gamma)=\left\{f \in E(\Gamma) \mid \operatorname{supp} f \subset \mathbf{T} \Gamma^{0}\right\},
$$

where $\mathbf{T} \Gamma^{0}$ denotes the isotropy group bundle of $\Gamma$. 
$E(\Gamma)$ is then ${ }^{*}$-algebra with a distinguished abelian subalgebra $D(\Gamma)$. Further, $D(\Gamma) \cong C_{c}\left(\Gamma^{0}\right) . E(\Gamma)$ becomes a pre-Hilbert $D(\Gamma)$-module; the completion, which Kumjian denotes by $\mathcal{H}(\Gamma)$, is a Hilbert $C_{0}\left(\Gamma^{0}\right)$-module.

Kumjian then constructs a ${ }^{*}$-homomorphism $\pi: E(\Gamma) \longrightarrow L(\mathcal{H}(\Gamma))$ such that $\pi(f) g=f g$ (the convolution product) for all $f, g \in E(\Gamma)$ and defines $A(\Gamma)$ to be the closure of $\pi(E(\Gamma))$ and $B(\Gamma)$ to be the closure of $\pi(D(\Gamma))$. It turns out that $B(\Gamma)$ is diagonal in $A(\Gamma)$, and this shows that every twist gives rise to a diagonal pair of $C^{*}$-algebras.

Conversely, given $C^{*}$-algebras $A$ and $B$ with $B$ diagonal in $A$, Kumjian constructs a twist $\Gamma$ such that $A \cong A(\Gamma)$ and $B \cong B(\Gamma)$, giving a bijective correspondence between twists and diagonal pairs.

The following theorem is perhaps known to experts, but we could not find it in the literature.

Theorem 2. Let $\mathcal{G}$ be a Hausdorff, amenable, $r$-discrete equivalence relation. Then $\left(C^{*}(\mathcal{G}), C_{0}\left(\mathcal{G}^{0}\right)\right)$ is a diagonal pair in Kumjian's sense, and the associated twist is the trivial twist over $\mathcal{G}$.

Proof. Given $\mathcal{G}$, we construct the trivial twist $\Gamma_{1}$ over $\mathcal{G}$. That is, let $\Gamma_{1}=\mathcal{G} \times \mathbf{T}$. The T-groupoid operations on $\Gamma_{1}$ are as follows:

- $\left(g_{1}, t_{1}\right)\left(g_{2}, t_{2}\right)=\left(g_{1} g_{2}, t_{1} t_{2}\right)$;

- $(g, t)^{-1}=\left(g^{-1}, \bar{t}\right)$;

- $s(g, t)=(g, t)^{-1}(g, t)=(s(g), 1)$ (hence we identify the unit space of $\Gamma_{1}$ with the unit space of $\mathcal{G})$;

- $t_{1}\left(g, t_{2}\right)=\left(g, t_{1} t_{2}\right)$.

Note that $\mathcal{G}$ can be viewed as a subgroupoid of $\Gamma_{1}$ via $g \mapsto(g, 1)$. Thus, given a continuous equivariant function $f$ with compact support in $\Gamma_{1}$ (i.e. an element of Kumjian's $E\left(\Gamma_{1}\right)$ ), we can define $\hat{f} \in C_{c}(\mathcal{G})$ by $\hat{f}=\left.f\right|_{\mathcal{G}}$. It is easily checked that $\stackrel{\wedge}{ }:\left(\Gamma_{1}\right) \longrightarrow C_{c}(\mathcal{G})$ is an isomorphism of *algebras.

Thus, since $C_{c}(\mathcal{G})$ is dense in both $A\left(\Gamma_{1}\right)$ and $C^{*}(\mathcal{G})$, in order to see that $A\left(\Gamma_{1}\right) \cong$ $C^{*}(\mathcal{G})$, it suffices to show:

$$
\|f\|_{A\left(\Gamma_{1}\right)}=\|f\|_{C^{*}(\mathcal{G})} \quad \text { for every } f \in C_{c}(\mathcal{G}) .
$$

Because $\mathcal{G}$ is amenable, $C^{*}(\mathcal{G})$ coincides with $C_{r}^{*}(\mathcal{G})$ [1, Proposition 6.1.5], so $\|f\|_{C^{*}(\mathcal{G})}=\sup _{u \in \mathcal{G}^{0}}\left\|\operatorname{Ind} \epsilon_{u}(f)\right\|$, where $\operatorname{Ind} \epsilon_{u}$ is the representation of $C_{c}(\mathcal{G})$ on $L^{2}\left(\nu_{u}^{-1}\right)$ given by $\operatorname{Ind} \epsilon_{u}(f) \xi(x)=\int f(y) \xi\left(y^{-1} x\right) d \lambda^{r(x)}(y)$. Here, $\nu_{u}$ denotes the measure on $\mathcal{G}$ induced by the measure $\epsilon_{u}$ on $\mathcal{G}^{0}$, given by $\nu_{u}=\int_{v \in \mathcal{G}^{0}} \lambda^{v} d \epsilon_{u}(v)$. The measure $\nu_{u}^{-1}$ is given by $\int \phi(x) d \nu_{u}^{-1}(x)=\int \phi\left(x^{-1}\right) d \nu_{u}(x)$.

Now, for $u \in \mathcal{G}^{0}$, consider the representation $\delta_{u}: C_{0}\left(\mathcal{G}^{0}\right) \longrightarrow \mathbf{C}$ given by $\delta_{u}(f)=$ $f(u)$. Since $\mathcal{H}\left(\Gamma_{1}\right)$ is a (right) Hilbert $C_{0}\left(\mathcal{G}^{0}\right)$-module with a left action of $A\left(\Gamma_{1}\right)$ by adjointable operators, we can induce the representation $\delta_{u}$ up to a representation Ind $\delta_{u}$ of $A\left(\Gamma_{1}\right)$ on the Hilbert space $\mathcal{H}\left(\Gamma_{1}\right) \otimes_{C_{0}\left(\mathcal{G}^{0}\right)}$ C. We claim that for every $u \in \mathcal{G}^{0}, \operatorname{Ind} \delta_{u}$ is unitarily equivalent to $\operatorname{Ind} \epsilon_{u}$. 
Define $v: \mathcal{H}\left(\Gamma_{1}\right) \odot \mathbf{C} \longrightarrow L^{2}\left(\nu_{u}^{-1}\right)$ by $v\left(\sum g_{i} \otimes \lambda_{i}\right)=\sum \lambda_{i} g_{i}$. We have

$$
\begin{aligned}
\left\langle\sum_{i} g_{i} \otimes \lambda_{i}, \sum_{j} h_{j} \otimes \mu_{j}\right\rangle_{\mathcal{H}\left(\Gamma_{1}\right) \otimes \mathbf{C}} & =\sum_{i, j}\left\langle g_{i} \otimes \lambda_{i}, h_{j} \otimes \mu_{j}\right\rangle \\
& =\sum_{i, j} \overline{\lambda_{i}} \mu_{j} g_{i}^{*} h_{j}(u) \\
& =\sum_{x \in \mathcal{G}^{u}} \sum_{i, j} \overline{\lambda_{i}} \mu_{j} \overline{g_{i}\left(x^{-1}\right)} h_{j}\left(x^{-1}\right) \\
& =\sum_{x \in \mathcal{G}^{u}} \overline{v\left(\sum_{i} g_{i} \otimes \lambda_{i}\right)\left(x^{-1}\right)} v\left(\sum_{j} h_{j} \otimes \mu_{j}\right)\left(x^{-1}\right) \\
& =\left\langle v\left(\sum_{i} g_{i} \otimes \lambda_{i}\right), v\left(\sum_{j} h_{j} \otimes \mu_{j}\right)\right\rangle_{L^{2}\left(\nu_{u}^{-1}\right)} .
\end{aligned}
$$

Thus $v$ can be extended to a unitary (also denoted by $v$ ) from $\mathcal{H}\left(\Gamma_{1}\right) \otimes_{C_{0}\left(\mathcal{G}^{0}\right)} \mathbf{C}$ to $L^{2}\left(\nu_{u}^{-1}\right)$. Also, for all $f \in C_{c}(\mathcal{G})$,

$$
\begin{aligned}
v \operatorname{Ind} \delta_{u}(f)\left(\sum g_{i} \otimes \lambda_{i}\right) & =v\left(\sum f g_{i} \otimes \lambda_{i}\right) \\
& =\sum \lambda_{i} f g_{i} \\
& =\sum \lambda_{i} \operatorname{Ind} \epsilon_{u}(f) g_{i} \\
& =\operatorname{Ind} \epsilon_{u}(f) v\left(\sum g_{i} \otimes \lambda_{i}\right) .
\end{aligned}
$$

Thus Ind $\delta_{u}$ is unitarily equivalent to Ind $\epsilon_{u}$ for every $u \in \mathcal{G}^{0}$.

Since $\left\{\delta_{u}\right\}$ is a separating family of representations of $C_{0}\left(\mathcal{G}^{0}\right),\left\{\operatorname{Ind} \delta_{u}\right\}$ is a separating family of representations of $A\left(\Gamma_{1}\right)$. Thus, for $f \in C_{c}(\mathcal{G})$,

$$
\|f\|_{A\left(\Gamma_{1}\right)}=\sup _{u \in \mathcal{G}^{0}}\left\|\operatorname{Ind} \delta_{u}(f)\right\|=\sup _{u \in \mathcal{G}^{0}}\left\|\operatorname{Ind} \epsilon_{u}(f)\right\|=\|f\|_{C^{*}(\mathcal{G})},
$$

as desired.

Now, by [4, Theorem 2.9], it follows that $C_{0}\left(\mathcal{G}^{0}\right)$ is diagonal in $C^{*}(\mathcal{G})$. Since the correspondence between diagonal pairs and twists is bijective (up to isomorphism), we must have that $\Gamma$, the twist constructed from this diagonal pair, is isomorphic to $\Gamma_{1}$, the trivial twist over $\mathcal{G}$.

Corollary 1. Every AF-algebra contains a subalgebra which is diagonal in Kumjian's sense.

Proof. By Theorem 1, every AF-algebra $A$ is the $C^{*}$-algebra of a Hausdorff, $r$ discrete equivalence relation, which is amenable by [6, Corollary 5.5]. Thus, by Theorem 2, $A$ contains a diagonal subalgebra.

In [9], Strătilă and Voiculescu defined a notion of a diagonal subalgebra for AFalgebras, and it is not surprising that the two notions of diagonal coincide in the $\mathrm{AF}$ case. We briefly review the Strătilă-Voiculescu set-up.

Given a unital AF-algebra $A=\overline{\bigcup_{n=0}^{\infty} A_{n}}$, where it is assumed that $A_{0} \cong \mathbf{C}$, Strătilă and Voiculescu inductively define an ascending sequence of abelian $C^{*}$ subalgebras $\left\{C_{n}\right\}$ as follows:

- $C_{0}=A_{0}$;

- given $C_{n}$, define $C_{n+1}=\operatorname{span} C_{n} D_{n+1}$, where $D_{n+1}$ is an arbitrary maximal abelian self-adjoint subalgebra of $A_{n+1} \cap A_{n}^{\prime}$. 
The diagonal subalgebra $C$ is defined to be the closure of the union of the $C_{n}$ 's.

Strătilă and Voiculescu's AF masa led to Renault's notion of Cartan subalgebra which led in turn to Kumjian's notion of diagonal. The following result is similar to [8, III.1.15] and is also related to [4, 6.16]. In [9], only unital AF-algebras were considered, but the definition of $C$ makes sense in the nonunital case, and it is in that more general setting that the following theorem is proved.

Theorem 3. Let $A$ be an AF-algebra. Then Strătilă and Voiculescu's subalgebra $C$ is diagonal in Kumjian's sense.

Proof. In light of Theorem1, we can view $A$ as $C^{*}\left(\left.\mathcal{G}_{E}\right|_{S}\right)$ for some Bratteli diagram $E$ for $A$ and some subset $S$ of vertices of $E$. We claim that the $D_{n}$ 's can be chosen such that for each $n, C_{n}=\operatorname{span}\left\{1_{Z(\alpha, \alpha)} \mid r(\alpha) \in V^{n}\right\}$ (recall that $V^{n}$ denotes the $n$th level of vertices of the Bratteli diagram).

Suppose $C_{0}, C_{1}, \ldots, C_{n}$ are as in the above claim. For each edge $e$ with $r(e) \in$ $V^{n+1}$, define $f_{e}=\sum 1_{Z(\alpha, \alpha)}$, where the sum runs over all paths $\alpha$ whose last edge is $e$ (we also make the implicit assumption, here and from now on, that all paths start in the distinguished set $S$ ). Also, for every $v \in V^{n+1} \cap S$, define $f_{v}=1_{Z(v, v)}$. Now define

$$
D_{n+1}=\operatorname{span}\left\{f_{e}, f_{v} \mid r(e) \in V^{n+1}, v \in V^{n+1} \cap S\right\} .
$$

Lemma 2. $D_{n+1}$ is a MASA in $A_{n+1} \cap A_{n}^{\prime}$.

Proof. Clearly $D_{n+1}$ is abelian. To show that it is a MASA in $A_{n+1} \cap A_{n}^{\prime}$, we will show that $x \in A_{n+1} \cap A_{n}^{\prime} \cap D_{n+1}^{\prime}$ implies $x \in D_{n+1}$. So fix $x \in A_{n+1} \cap A_{n}^{\prime} \cap D_{n+1}^{\prime}$. We can write $x=\sum \lambda_{\alpha, \beta} 1_{Z(\alpha, \beta)}$, where the sum runs over all $\alpha, \beta$ with $r(\alpha)=$ $r(\beta) \in V^{n+1}$ and each pair appears only once.

First suppose that there exist $\alpha_{0} \neq \beta_{0}$ such that $\lambda_{\alpha_{0}, \beta_{0}} \neq 0$. Then one of the following three cases holds:

Case 1: One of $\alpha_{0}$ or $\beta_{0}$ is a zero-length path.

Case 2: $\alpha_{0}$ and $\beta_{0}$ have the same last edge (hence differ somewhere before the last edge).

Case 3: $\alpha_{0}$ and $\beta_{0}$ have different last edges.

For Case 1, assume (without loss of generality) that $\left|\alpha_{0}\right|=0$ and let $y=$ $1_{Z\left(\alpha_{0}, \alpha_{0}\right)} \in D_{n+1}$. For Case 2, write $\alpha_{0}=\gamma_{0} e$ where $e$ is an edge, and let $y=1_{Z\left(\gamma_{0}, \gamma_{0}\right)} \in A_{n}$. For Case 3, denote by $e_{0}$ the last edge of $\alpha_{0}$, and let $y=f_{e_{0}} \in D_{n+1}$.

In all three cases, $x$ and $y$ should commute, but the reader may tediously verify that $1_{Z\left(\beta_{0}, \alpha_{0}\right)} x y 1_{Z\left(\beta_{0}, \alpha_{0}\right)}=0$ and $1_{Z\left(\beta_{0}, \alpha_{0}\right)} y x 1_{Z\left(\beta_{0}, \alpha_{0}\right)}=\lambda_{\alpha_{0}, \beta_{0}} 1_{Z\left(\beta_{0}, \alpha_{0}\right)} \neq 0$. Thus $x$ has no "off-diagonal" entries. So we can write $x=\sum \lambda_{\alpha} 1_{Z(\alpha, \alpha)}$.

Now suppose there exist $\alpha_{1} \neq \alpha_{2}$ such that $\alpha_{1}, \alpha_{2}$ have the same last edge, but $\lambda_{\alpha_{1}} \neq \lambda_{\alpha_{2}}$. For $i=1,2$, write $\alpha_{i}=\gamma_{i} e$ where $e$ is an edge, and set $y=$ $1_{Z\left(\gamma_{1}, \gamma_{2}\right)} \in A_{n}$. The reader may check that $1_{Z\left(\alpha_{1}, \alpha_{1}\right)} x y \neq 1_{Z\left(\alpha_{1}, \alpha_{1}\right)} y x$. Hence $x y \neq y x$, violating the assumption that $x \in A_{n}^{\prime}$.

Thus $x=\sum \lambda_{\alpha} 1_{Z(\alpha, \alpha)} \in A_{n+1} \cap A_{n}^{\prime} \cap D_{n+1}^{\prime}$ implies that $\lambda_{\alpha_{1}}=\lambda_{\alpha_{2}}$ whenever the last edges of $\alpha_{1}$ and $\alpha_{2}$ agree, which implies that $x \in D_{n+1}$.

Now, $C_{n+1}=\operatorname{span} C_{n} D_{n+1} \subset \operatorname{span}\left\{1_{Z(\alpha, \alpha)} \mid r(\alpha) \in V^{n+1}\right\}$. To show the reverse inclusion, fix $\alpha$ with $r(\alpha) \in V^{n+1}$. If $|\alpha|=0,1_{Z(\alpha, \alpha)} \in D_{n+1} \subset C_{n+1}$. If not, write $\alpha=\gamma e$ where $e$ is an edge and note that $1_{Z(\alpha, \alpha)}=f_{e} 1_{Z(\gamma, \gamma)} \in \operatorname{span}\left\{C_{n}, D_{n+1}\right\}$. 
Thus, with the $D_{n}$ 's chosen in this way, $C_{n}=\operatorname{span}\left\{1_{Z(\alpha, \alpha)} \mid r(\alpha) \in V^{n}\right\}$ for every $n$, so $C=\overline{\operatorname{span}}\left\{1_{Z(\alpha, \alpha)}\right\}=C_{0}\left(\left(\left.\mathcal{G}_{E}\right|_{S}\right)^{0}\right)$, which is diagonal in Kumjian's sense.

Now we have shown that the $C_{n}$ 's can be chosen in such a way that $C$ is diagonal. Now, suppose $\tilde{C}$ is another Strătilă-Voiculescu diagonal. Then there exists an automorphism $\alpha$ of $A$ which maps $C$ to $\tilde{C}$. In fact, this automorphism can be chosen to be approximately inner (i.e. there exists a sequence $\left\{u_{k}\right\}$ of unitaries in $A$ such that $\alpha(a)=\lim _{k \rightarrow \infty} u_{k} a u_{k}^{*}$; see [7]). Defining $\tilde{P}=\alpha \circ P \circ \alpha^{-1}$, the reader may check that $\tilde{P}$ is a faithful conditional expectation from $A$ onto $\tilde{C}$, and that ker $\tilde{P}=\alpha(\operatorname{ker} P)=\overline{\operatorname{span}} N_{f}(\tilde{C})$. Thus $\tilde{C}$ is diagonal in Kumjian's sense.

\section{REFERENCES}

1. C. Anantharaman-Delaroche and J. Renault, Amenable groupoids, Mem. Amer. Math. Soc., (to appear).

2. O. Bratteli, Inductive limits of finite dimensional $C^{*}$-algebras, Trans. Amer. Math. Soc. 171 (1972), 195-234. MR 47:844

3. E. Effros, Dimensions and $C^{*}$-algebras, CBMS Regional Conf. Ser. in Math. no. 46, American Mathematical Society, Providence, RI, 1980. MR 84k:46042

4. A. Kumjian, On $C^{*}$-diagonals, Can. J. Math. 38 (1986), 969-1008. MR 88a:46060

5. A. Kumjian, D. Pask, and I. Raeburn, Cuntz-Krieger algebras of directed graphs, Pacific J. Math. 184 (1998), 161-174. CMP 98:13

6. A. Kumjian, D. Pask, I. Raeburn, and J. Renault, Graphs, groupoids, and Cuntz-Krieger algebras, J. Funct. Anal. 144 (1997), 505-541. MR 98g:46083

7. S.C. Power, Limit algebras, Pitman Research Notes in Mathematics, vol. 278, Longman Scientific and Technical, London, 1992. MR 94g:46001

8. J. N. Renault, A groupoid approach to $C^{*}$-algebras, Lecture Notes in Math., no. 793, SpringerVerlag, New York, 1980. MR 92h:46075

9. S. Strătilă and D. Voiculescu, Representations of AF-algebras and of the group $U(\infty)$, Lecture Notes in Math., no. 486, Springer, New York, 1975. MR 56:16391

Department of Mathematics, Arizona State University, Tempe, Arizona 85287

E-mail address: Drinen@asu.edu 